\title{
The value of diffusion-weighted MRI (DW-MRI) as a tool to differentiate between various tumors and inflammatory diseases in the head and neck region
}

\author{
Alan A Peters ${ }^{1 \# *}$, Soung Y Kim ${ }^{1 \#}$, Peter Vermathen ${ }^{2}$, Harriet C Thoeny ${ }^{3,4}$ and Dechen W Tshering Vogel ${ }^{1}$ \\ ${ }^{1}$ Department of Diagnostic, Interventional and Pediatric Radiology, Inselspital, University of Bern, Freiburgstrasse 8, CH-3010 Bern, Switzerland \\ ${ }^{2}$ Magnetic Resonance Spectroscopy and Methodology - Department of Clinical Research, University of Bern, Erlachstrasse 9A, CH-3012 Bern, Switzerland \\ ${ }^{3}$ HFR Hôpital Fribourgois, University of Fribourg, CH-1708 Fribourg, Switzerland \\ ${ }^{4}$ Department of Urology, Inselspital, University of Bern, CH-3010 Bern, Switzerland \\ \#These authors contributed equally to this work
}

\begin{abstract}
Objective: To retrospectively determine whether diffusion-weighted magnetic resonance imaging (DW-MRI) can help in differentiating between various tumors and inflammatory lesions in the head and neck.

Materials/ methods: After obtaining ethics committee approval, a search was made in the radiology records for patients with diffusion- weighted MR imaging for inflammatory, benign and malignant lesions in the head and neck region, which were confirmed either by histopathology or by follow up for at least one year. Seventytwo patients were included in this study. The lesions were grouped into malignant lesions, comprising primary carcinomas ( $\mathrm{n}=17)$, metastasis $(\mathrm{n}=12)$ and lymphoma $(n=12)$, benign tumors $(n=24)$, inflammation $(n=4)$ and miscellaneous benign lesions $(n=3)$. Diffusion was assessed qualitatively by visual analysis and quantitatively by measuring the apparent diffusion coefficient (ADC) values.
\end{abstract}

Results: Benign lesions had higher ADC values than malignant lesions: The mean value for benign tumors was $1.46 \mathrm{x} 10^{-3} \mathrm{~mm} / \mathrm{sec}$ and for inflammatory lesions $1.51 \times 10^{-3} \mathrm{~mm}^{2} / \mathrm{sec}$. Lymphomas showed a mean value of $0.56 \times 10^{-3} \mathrm{~mm}^{2} / \mathrm{sec}$. The values for carcinoma and metastasis were similar with $0.84 \times 10^{-3} \mathrm{~mm} / \mathrm{sec}^{2}$ and $0.81 \times 10^{-3} \mathrm{~mm}^{2} / \mathrm{sec}$ respectively.

According to receiver operating characteristic (ROC) analysis, values higher than $1.03 \times 10^{-3} \mathrm{~mm}^{2} / \mathrm{sec}$ were ideal for differentiation between benign lesions and malignant tumors with a sensitivity of $88 \%$ and specificity of $79 \%$. Lymphomas had significantly lower ADC values than all other entities.

Conclusion: DW-MRI can help in differentiation of inflammatory and benign lesions from malignant lesions of the head and neck.

\begin{abstract}
Abbreviations: ADC: Apparent diffusion coefficient; AUC: Area under the curve; CT: Computed tomography; DW-MRI: Diffusionweighted magnetic resonance imaging; MRI: Magnetic resonance imaging; ROC: Receiver operating characteristics; SCC: Squamous cell carcinoma; T1-w: T1-weighted; T2-w: T2-weighted.
\end{abstract}

\section{Introduction}

Diffusion-weighted magnetic resonance imaging (DW-MRI), a functional MR imaging technique, is widely used for detection of acute stroke, myocardial ischemia, assessing different tumors (like liver, kidneys or prostate) and for assessing organ function and small bowel pathologies [1-7]. In the head and neck region, DW-MRI has been used for characterization of primary tumors, nodal metastasis, prediction and monitoring of treatment response, for differentiation between recurrent tumors and post-therapeutic changes or to study salivary gland function [8-11].

The radiological assessment of the head and neck area can be challenging due to its complex anatomy. Changes caused by tumors and inflammation can appear very similar, making it sometimes difficult to differentiate between them, especially if clinical signs of infection such as fever and accompanying changes in blood parameters are absent, as in many subacute and chronic infections or in granulomatous diseases. Tumors can also show infiltrative growth into the surrounding tissue, mimicking inflammation and a biopsy is often required for the diagnosis. The use of a non-invasive tool such as DW-MRI, if proven to be useful in this matter, can lead to a reduction in the number of biopsies required and also pinpoint suspicious areas where biopsies should be taken to reduce false negative results.

Many publications show the benefits of DW-MRI in the differentiation between benign and malignant tumors, for detecting lymph node metastases and tumor recurrence [12-16] and for evaluation of inflammation in different areas of the body $[17,18]$.

${ }^{\star}$ Correspondence to: Alan A Peters, Department of Radiology, Neuroradiology and Nuclear Medicine, Inselspital, University of Bern, Freiburgstrasse 8, CH3010 Bern, Switzerland, Tel: (0041)632/6575; E-mail: Alan.Peters@insel.ch

Key words: diffusion-weighted imaging, head and neck, inflammation, benign tumors, carcinoma, metastases, lymphoma

Received: November 23, 2018; Accepted: December 03, 2018; Published: December 11, 2018 
Peters AA (2018) The value of diffusion-weighted MRI (DW-MRI) as a tool to differentiate between various tumors and inflammatory diseases in the head and neck region

However, there are limited studies evaluating inflammatory lesions in the head and neck region with DW-MRI [19-21], probably because inflammatory conditions are mostly only imaged when complications are suspected, and contrast-enhanced computed tomography (CT) is still more commonly used for this purpose.

Malignant lesions are usually hypercellular and show diffusion restriction with resulting lower apparent diffusion coefficient (ADC) values compared to benign lesions, which are less cellular. Inflammatory lesions are edematous and relatively hypocellular, which should allow more diffusion and therefore higher ADC values. We hypothesize, that these lesions can be differentiated with DW-MRI. The purpose of this study is to find out retrospectively whether quantitative and qualitative assessment of DW-MRI can help in the differentiation between various malignant and benign lesions (including inflammatory lesions) in the head and neck, and to find the optimal threshold ADC value that best allows this differentiation.

\section{Materials and methods}

\section{Patients}

This is a retrospective study approved by the institutional review board; consent was waived because of the retrospective nature of the study. A search was made in the radiological database of our hospital (Centricity RIS-I, GE Healthcare, Milwaukee, Wisconsin, USA) and all patients with tumors or inflammatory changes, who had diagnostic quality MR examinations, including diffusion-weighted sequences of the neck, from the beginning of 2007 to the end of 2013, were included in the study. The benign or malignant nature of the lesions was proven either with histopathology after surgery or biopsy or after a follow up of at least one year for benign lesions. This search resulted in 72 patients (24 females, 48 males, age range: 16-95 years, mean age: 58.8 years), who were all included in the present study (supplementary Table 1).

\section{MR imaging}

MR imaging was performed at 1.5T (Magnetom AERA, Siemens Healthcare, Erlangen, Germany) with a standard receive-only head and neck coil. Due to the different clinical presentations of the patients, different imaging protocols were used and so the sequences varied. However, all included at least the following sequences:

A coronal turbo inversion recovery sequence (voxel size: $1.0 \times 1.0$ x $4.0 \mathrm{~mm}$, FoV read: $260 \mathrm{~mm}$, slice thickness: $4.0 \mathrm{~mm}$, repetition time / echo time: $4000 \mathrm{~ms} / 48 \mathrm{~ms}$ ), an axial T1-weighted (T1-w) sequence ( $0.3 \times 0.3 \times 3.0 \mathrm{~mm}, 220 \mathrm{~mm}, 3.0 \mathrm{~mm}, 580 \mathrm{~ms} / 12 \mathrm{~ms})$, an axial T2-w sequence $(0.6 \times 0.6 \times 3.0 \mathrm{~mm}, 220 \mathrm{~mm}, 3.0 \mathrm{~mm}, 4000 \mathrm{~ms} / 84 \mathrm{~ms})$ and DW-images in the axial plane $(1.3 \times 1.3 \times 4.0 \mathrm{~mm}, 250 \mathrm{~mm}, 4.0 \mathrm{~mm}$, $3000 \mathrm{~ms} / 111 \mathrm{~ms})$. After injection of contrast media, T1-w fat-saturated sequences in the axial plane $(0.4 \times 0.4 \times 3.0 \mathrm{~mm}, 220 \mathrm{~mm}, 3.0 \mathrm{~mm}, 533$ $\mathrm{ms} / 12 \mathrm{~ms})$ and in the coronal plane $(0.5 \times 0.5 \times 3.0 \mathrm{~mm}, 300 \mathrm{~mm}, 3.0$ $\mathrm{mm}, 509 \mathrm{~ms} / 9.6 \mathrm{~ms}$ ) were acquired. Contrast agents used were either Gadovist (Bayer, Zürich, Switzerland) for patients with normal kidney function at a dose of $0.1 \mathrm{ml}$ per kilogram body weight or Dotarem (Bracco SpA, Milano, Italy) for patients with impaired kidney function at a dose of $0.2 \mathrm{ml}$ per kilogram body weight.

A minimum of two $b$-values to a maximum of six b-values were used with b-values ranging from $0,50,100,150,300,400,500,600$, 800,900 and $1000 \mathrm{~mm} 2 / \mathrm{sec}$ depending on the MR protocol used. The highest b-value of each study was used to calculate the ADC (either 800 or $1000 \mathrm{~mm}^{2} / \mathrm{sec}$ ).

\section{Image analysis}

Images were analyzed qualitatively and quantitatively in consensus, by two radiologists with 20 years' (HT) respectively with 13 years' (DWT) experience in MR imaging of the head and neck, blinded to the final diagnosis. The signal intensity of the lesions on $\mathrm{T} 1-\mathrm{w}$ and T2-w sequences relative to the adjacent skeletal muscle and presence of contrast enhancement were also documented.

Qualitative analysis was performed by visual assessment of the high b-value images and the corresponding ADC maps in conjunction with the morphological images. Diffusion restriction was present when there was a high signal in the high b-value image with corresponding low signal on the ADC map. High signal on the high b-value images with high value in the corresponding ADC map was interpreted as T2shine-through and not as diffusion restriction. Low signal on both the high b-value images and ADC maps was interpreted as an absence of diffusion restriction.

Quantitative analysis consisted of ADC value measurement. Maps of the ADC were automatically obtained from the diffusion-weighted images by use of a multiple point regression method using the following equation: $\mathrm{I}_{\mathrm{b}}=\mathrm{I}_{0} \times \exp (-\mathrm{b} \times A D C)$, where $\mathrm{I}_{0}$ and $\mathrm{I}_{\mathrm{b}}$ are the signal intensity value of each pixel in a region of interest at a b-value of $0 \mathrm{sec} / \mathrm{mm}^{2}$ respectively $\mathrm{b} \mathrm{sec} / \mathrm{mm}^{2}$. The ADC values were measured by placing a circular region of interest (ROI) in each area of pathological change. While placing the ROI in the lesions, areas with obvious contrast enhancement in the corresponding T1w-sequences were targeted to exclusively include solid tumor mass and avoid areas of necrosis.

\section{Histopathology}

Imaging results were compared to histopathological results and / or follow-up exams. In sixty-two patients, including all the patients with malignancies, histopathological correlation for the lesions were available (19 surgeries and 43 biopsies). The diagnoses in the remaining ten patients with benign tumors, inflammatory or miscellaneous lesions were confirmed by a follow-up examination after at least one year.

\section{Statistical analysis}

A power analysis with the current values, i.e. 41 malignant lesions and 31 benign lesions, including benign tumors, inflammatory and miscellaneous lesions, and a standard deviation of approximately 0.2 $(13 \%)$, taken from the literature $(22,23)$, yields that an ADC difference of 0.14 could be detected at a significance level of 0.05 and a statistical power of 0.8 . In view of previously detected ADC differences, the sample size was therefore considered sufficient.

Statistical analysis was performed by use of a Kruskal-Wallis test to find out whether there were any significant differences in ADC values between the groups. In a second step the groups were compared pairwise by use of the Mann-Whitney- $U$ test to quantify statistical differences.

All statistical tests were two-sided, a p-value of less than 0.05 was considered statistically significant after corrections for multiple comparisons. Box-whisker plots were used to visualize group-wise differences in quantitative diffusion parameters. Optimal threshold values to discriminate between the groups were determined using receiver operating characteristics (ROC) curves, attributing equal weight to sensitivity and specificity. The value that corresponded with the ROC-curve nearest to the top left-hand corner was considered the 
Peters AA (2018) The value of diffusion-weighted MRI (DW-MRI) as a tool to differentiate between various tumors and inflammatory diseases in the head and neck region

optimal threshold-value. Statistical analysis was performed with SPSS, version 12.0.1 (SPSS, Chicago, IL, USA) and Excel 2011 (Microsoft, Redmond, WA, USA).

\section{Results}

Out of the 72 patients included in this study, 41 patients had malignant lesions (57\%) and 31 had benign lesions (43\%). Malignant lesions included primary carcinomas $(\mathrm{n}=17)$, lymphomas $(\mathrm{n}=12)$ and cervical metastases $(n=12)$.

Primary carcinomas were located in the oropharynx $(n=6)$, the nasopharynx $(n=4)$, the hypopharynx $(n=3)$, in the parotid gland $(n=2)$, the masticator space $(n=1)$ or the thyroid gland $(n=1)$ and consisted of SCC $(n=11)$, undifferentiated nasopharyngeal carcinoma $(\mathrm{n}=2)$, adenocarcinoma $(\mathrm{n}=2)$ and papillary carcinoma of the thyroid gland $(\mathrm{n}=2)$.

Cervical metastases originated from various tumors: SCC of the oropharynx $(n=4)$, the larynx $(n=2)$, adenocarcinoma of the parotid gland $(n=1)$, nasopharyngeal carcinoma of the maxillary sinus $(n=1)$, neuroendocrine tumor of the thymus $(n=1)$, infratemporal melanoma $(n=1)$, parapharyngeal synovial cell sarcoma $(n=1)$ and from retropharyngeal malignant nerve sheath tumor $(n=1)$.

Benign lesions consisted of benign tumors $(n=24)$, inflammation $(n=4)$ and benign miscellaneous lesions consisting of sarcoidosis $(n=1)$, Wegeners granulomatosis $(\mathrm{n}=1)$ and a case of Rosai-Dorfman disease $(\mathrm{n}=1)$.
Benign tumors included pleomorphic adenoma $(\mathrm{n}=11)$ hemangioma $(n=3)$, Warthin tumors $(n=2)$, fibrolipoma $(n=1)$, lipoma $(n=1)$, schwannoma $(n=1)$, meningioma $(n=1)$, glomus tumor $(n=1)$, odontogenic myxoma of the maxilla $(\mathrm{n}=1)$, lymphangioma $(\mathrm{n}=1)$ and a lymphoepithelial cyst $(\mathrm{n}=1)$. The inflammatory lesions included a case of myositis of the temporalis muscle $(n=1)$, an inflammatory pseudotumor of the pterygopalatine fossa $(n=1)$, focal parotitis $(n=1)$ and a diffuse inflammation of the cheek after dental extraction $(n=1)$.

\section{Image analysis}

The MRI appearances of the lesions are presented in Table 1 along with the results of the qualitative and quantitative analysis. Most lesions were hypointense compared to adjacent skeletal muscle on T1-w sequences and hyperintense on $\mathrm{T} 2-\mathrm{w}$ sequences with varying amounts of contrast enhancement as shown in the table.

Qualitative evaluation of DW-MRI showed that $40 / 41$ of malignant lesions had restricted diffusion. The only exception was a case of papillary carcinoma of the thyroid gland. Diffusion restriction was most marked in lymphoma (Figure 1).

Most benign tumors did not show any diffusion restriction (Figure 2). Diffusion restriction was present in schwannoma, meningioma and Warthin tumors. Focal areas of restricted diffusion were also seen in a cystic lymphangioma and one pleomorphic adenoma.

The inflammatory and benign miscellaneous lesions did not show any quantifiable diffusion restriction (Figure 3).

Table 1. Imaging appearance of the lesions with qualitative and quantitative evaluation of DW-MRI

\begin{tabular}{|c|c|c|c|c|c|c|c|c|c|}
\hline Pathology & & T1 & T2 & CE & High b-value & ADC map & DR & ADC range & ADC mean \\
\hline Malignant lesions & $=41)$ & & & & & & & & 0.85 \\
\hline Carcinoma & $\mathrm{n}=16$ & $\downarrow$ & $\uparrow$ & +++ & $\uparrow$ & $\downarrow$ & + & $0.39-1.30$ & 0.83 \\
\hline$(n=17)$ & $\mathrm{n}=1$ & $\downarrow$ & $\uparrow$ & +++ & $\uparrow$ & $\uparrow$ & - & I & 1.02 \\
\hline Metastasis $(\mathrm{n}=12)$ & & $\downarrow$ & $\uparrow$ & +++ & $\uparrow$ & $\downarrow$ & + & $0.42-1.41$ & 0.81 \\
\hline Lymphoma $(\mathrm{n}=12)$ & & $\downarrow$ & $\uparrow$ & +++ & $\uparrow$ & $\downarrow$ & + & $0.43-0.79$ & 0.56 \\
\hline Benign tumors ( $n$ & & & & & & & & & 1.46 \\
\hline Adenoma $(\mathrm{n}=11)$ & $\mathrm{n}=10$ & $\downarrow$ & $\uparrow$ & ++ & $\uparrow$ & $\uparrow$ & - & $1.14-2.25$ & 1.60 \\
\hline & $\mathrm{n}=1$ & $\downarrow$ & $\uparrow$ & ++ & $\uparrow$ & $\downarrow$ & + & 1 & 1.94 \\
\hline Hemangioma $(\mathrm{n}=3$ & & $\downarrow$ & $\uparrow$ & ++ & $\uparrow$ & $\uparrow$ & - & $1.14-2.26$ & 1.63 \\
\hline Warthin tumor $(\mathrm{n}=$ & & $\downarrow$ & $\downarrow$ & ++ & $\uparrow$ & $\downarrow$ & + & $0.60-0.78$ & 0.69 \\
\hline (Fibro)Lipoma (n= & & $\uparrow$ & $\uparrow$ & + & $\uparrow$ & $\uparrow$ & - & $0.84-1.67$ & 1.26 \\
\hline Schwannoma $(\mathrm{n}=1$ & & $\downarrow$ & $\uparrow$ & ++ & $\uparrow$ & $\downarrow$ & + & / & 1.60 \\
\hline Meningioma $(\mathrm{n}=1)$ & & $\downarrow$ & $\uparrow$ & ++ & $\uparrow$ & $\downarrow$ & + & I & 0.76 \\
\hline Glomus tumor $(\mathrm{n}=$ & & $\downarrow$ & $\uparrow$ & + & $\uparrow$ & $\uparrow$ & - & I & 1.24 \\
\hline Myxoma (n=1) & & $\downarrow$ & $\uparrow$ & + & $\uparrow$ & $\uparrow$ & - & / & 2.07 \\
\hline Lymphangioma (n & & $\downarrow$ & $\uparrow$ & + & $\uparrow$ & $\downarrow$ & + & / & 0.78 \\
\hline Lymphoepithelial & $(\mathrm{n}=1)$ & $\downarrow$ & $\uparrow$ & + & $\uparrow$ & $\uparrow$ & - & 1 & 2.37 \\
\hline Pathology & & T1 & T2 & $\mathbf{C E}$ & High b-value & ADC map & DR & ADC range & ADC mean \\
\hline Inflammatory lesi & $(n=4)$ & & & & & & & & 1.51 \\
\hline Myositis (n=1) & & $\downarrow$ & $\uparrow$ & +++ & $\uparrow$ & $\uparrow$ & - & I & 1.54 \\
\hline Inflammatory Pseu & umor $(n=1)$ & $\downarrow$ & $\uparrow$ & +++ & $\uparrow$ & $\uparrow$ & - & / & 1.51 \\
\hline Focal parotitis $(n=$ & & $\downarrow$ & $\uparrow$ & +++ & $\uparrow$ & $\uparrow$ & - & I & 1.04 \\
\hline Posttherapeutic inf & imation $(\mathrm{n}=1)$ & $\downarrow$ & $\uparrow$ & ++ & $\uparrow$ & $\uparrow$ & - & / & 1.97 \\
\hline Miscellaneous lesi & $s(n=3)$ & & & & & & & & 1.11 \\
\hline Wegeners disease & & $\uparrow$ & $\downarrow$ & +++ & $\uparrow$ & $\uparrow$ & - & I & 1.22 \\
\hline Sarcoidosis $(\mathrm{n}=1)$ & & $\downarrow$ & $\uparrow$ & +++ & $\uparrow$ & $\uparrow$ & - & I & 1.17 \\
\hline Rosai-dorfman-dis & $e(n=1)$ & $\downarrow$ & $\uparrow$ & + & $\uparrow$ & $\uparrow$ & - & / & 0.95 \\
\hline
\end{tabular}

- CE contrast enhancement; + mild, ++ moderate, +++ strong enhancement

- T1 (weighted); T2 (weighted); $\downarrow$ hypointense signal intensity (in comparison to adjacent muscle), $\uparrow$ hyperintense signal intensity (in comparison to adjacent muscle)

- DR diffusion restriction: - negative / + positive

- High b-value: image at $1000 \mathrm{~mm}^{2} / \mathrm{s}$ resp. $800 \mathrm{~mm}^{2} / \mathrm{s}$

- ADC unit: $x 10^{-3} \mathrm{~mm}^{2} / \mathrm{s}$ 
Peters AA (2018) The value of diffusion-weighted MRI (DW-MRI) as a tool to differentiate between various tumors and inflammatory diseases in the head and neck region
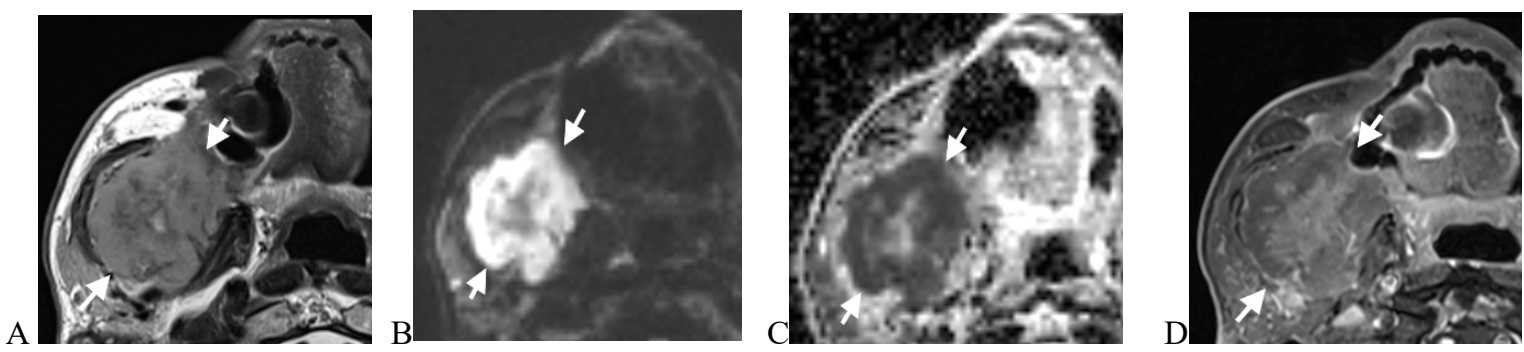

Figure 1. Axial MRI images of an 88-year-old male with lymphoma of the right mandible (arrows)

(A) T2-w image showing a lesion of the right mandible with intermediate signal intensity. (B) T1-w fat sat image with contrast media showing inhomogenous enhancement of the lesion. (C) High b-value DW-MR image showing high signal in the periphery with corresponding low signal on the ADC map. (D) The ADC value was $0.64 \times 10^{-3} \mathrm{~mm}^{2} / \mathrm{sec}$
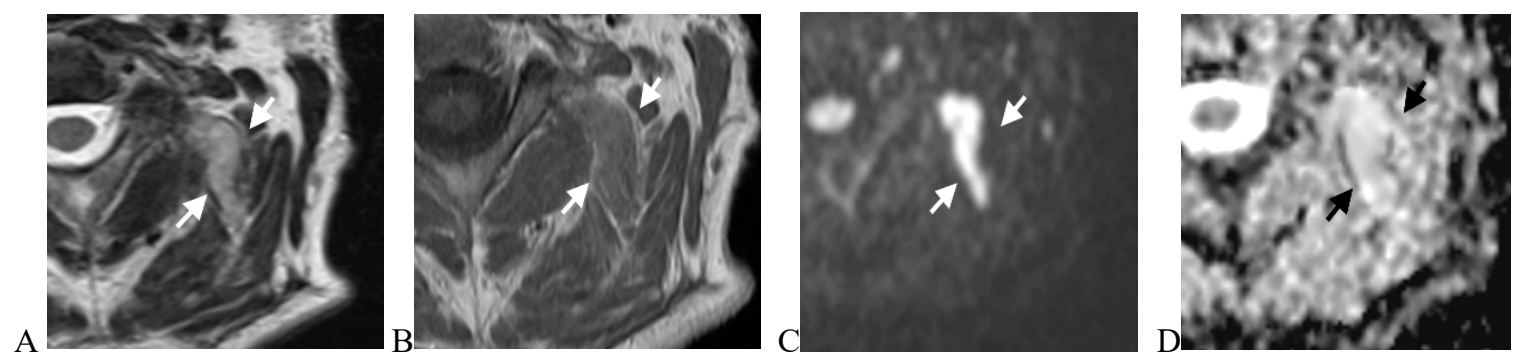

Figure 2. Axial MR images of a 52-year-old male with a fibrolipoma in the left-sided autochthonous muscles of the neck (arrows)

(A) T2-w image showing a slightly hyperintense lesion involving the adjacent muscles (arrows). (B) On the T1-w image, the lesion is slightly hyperintense to the adjacent muscles. (C) The high b-value image shows hyperintense signal, which remains high on the corresponding ADC map (D)
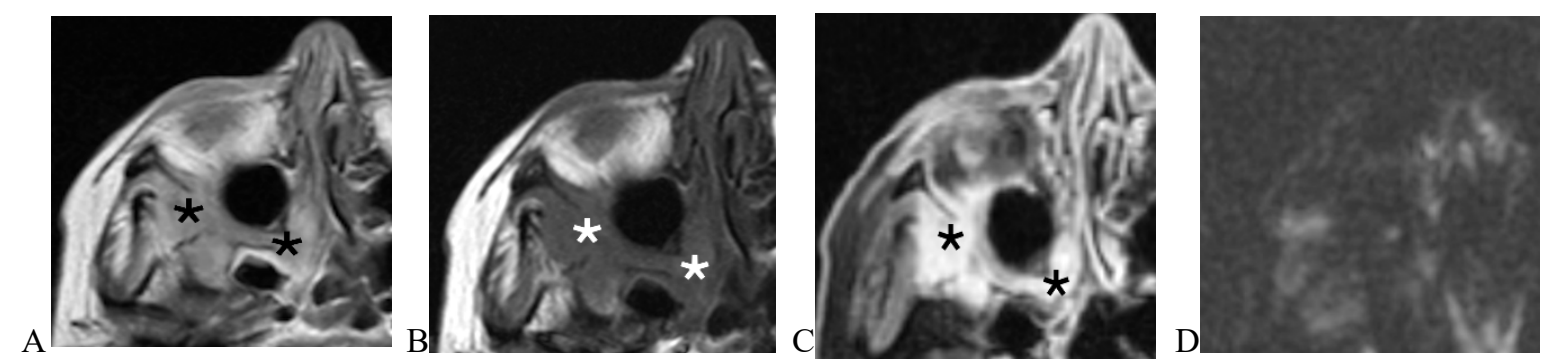

Figure 3. Axial MR images of a 62-year-old female patient with an inflammatory pseudotumor in the right pterygopalatine fossa and adjacent infratemporal fossa (asterisk) (A) T2-w image showing hyperintense signal in the right pterygopalatine and adjacent infratemporal fossa. (B) T1-w image before contrast shows low signal intensity in the same area and (C) T1-w fat sat image after injection of contrast media, which shows strong homogenous contrast enhancement. (D) High b-value DW-MR image showing absence of high signal intensity in the same area, indicating an absence of diffusion restriction

The sole use of qualitative analysis to detect diffusion restriction as an indicator of malignancy resulted in a sensitivity of $97.4 \%(40 /$ 41 malignant lesions showed restricted diffusion) and a specificity of $80.6 \%$ (6 / 31 benign lesions showed restricted diffusion).

Quantitative evaluation was based on the ADC values. Primary carcinomas had a mean ADC value of $(0.84 \pm 0.23$ [standard deviation] $)$ x $10^{-3} \mathrm{~mm}^{2} / \mathrm{sec}$ (range: $0.39-1.30 \times 10^{-3} \mathrm{~mm}^{2} / \mathrm{sec}$ ) and cervical metastases a mean value of $(0.81 \pm 0.27) \times 10^{-3} \mathrm{~mm}^{2} / \mathrm{sec}$ with a range of $0.42-1.4110^{-3} \mathrm{~mm}^{2} / \mathrm{sec}$. Lymphomas had a mean ADC value of $(0.56$ $\pm 0.1) \times 10^{-3} \mathrm{~mm}^{2} / \mathrm{sec}$ (range: $0.43-0.79 \times 10^{-3} \mathrm{~mm}^{2} / \mathrm{sec}$ ). Benign tumors showed a mean ADC value of $(1.46 \pm 0.52) \times 10^{-3} \mathrm{~mm}^{2} / \mathrm{sec}$ (range: 0.69 $-2.37 \times 10^{-3} \mathrm{~mm}^{2} / \mathrm{sec}$ ).

Inflammatory lesions showed a mean ADC value of $(1.51 \pm 0.38)$ x $10^{-3} \mathrm{~mm}^{2} / \mathrm{sec}$ (range: $1.04-1.97 \times 10^{-3} \mathrm{~mm}^{2} / \mathrm{sec}$ ). The results are presented graphically in Figure 4.

\section{Statistical analysis}

Comparing the different groups to one another, the MannWhitney-U test showed that benign tumors had significantly higher
ADC values compared to carcinomas $(\mathrm{p}=0.004)$, as well as to metastases $(\mathrm{p}=0.003)$ and to lymphomas ( $\mathrm{p}=0.00004)$.

Based on the ADC values, inflammation could be clearly differentiated from lymphomas after conservative Bonferroni correction for multiple comparisons $(p=0.036)$. Furthermore, there were only borderline significant differences $(p<0.06)$ between inflammation and carcinomas $(\mathrm{p}=0.055)$, as well as between inflammation and metastases $(\mathrm{p}=.053)$. Most likely this was due to the small sample size of inflammatory lesions $(n=4)$. There was no statistical significant difference between inflammatory lesions and benign tumors.

ROC analysis revealed the optimal threshold value to distinguish between benign lesions, including inflammatory and miscellaneous lesions, and malignant tumors to be $1.03 \times 10^{-3} \mathrm{~mm}^{2} / \mathrm{sec}$ with a sensitivity of $88 \%$ and a specificity of $79 \%$ for the ADC values (Figure $5 \mathrm{~A}$ ). The area under the curve (AUC) was 0.89 , suggesting a good quality of the analysis.

Primary carcinomas and cervical metastases in the neck area had similar ADC values, which is logical since both groups consist of the same tumor entities. 
Peters AA (2018) The value of diffusion-weighted MRI (DW-MRI) as a tool to differentiate between various tumors and inflammatory diseases in the head and neck region

ADC values of lymphomas were the lowest among all the lesions and there was a significant difference between the ADC values of lymphomas compared to primary carcinomas $(\mathrm{p}=0.009)$ and between lymphomas compared to metastases $(\mathrm{p}=0.047)$. According to the ROC analysis, a threshold value of $0.64 \times 10^{-3} \mathrm{~mm}^{2} / \mathrm{sec}$ was optimal for differentiating lymphomas from primary carcinomas with a sensitivity of $92 \%$ and a specificity of $88 \%$. The same threshold value could be used for differentiation between lymphomas and cervical metastases with a sensitivity of $92 \%$ and a specificity of $75 \%$ (Figure $5 b$ and $5 c$ ). The AUC for the ROC analysis between lymphoma and carcinoma was 0.87 and

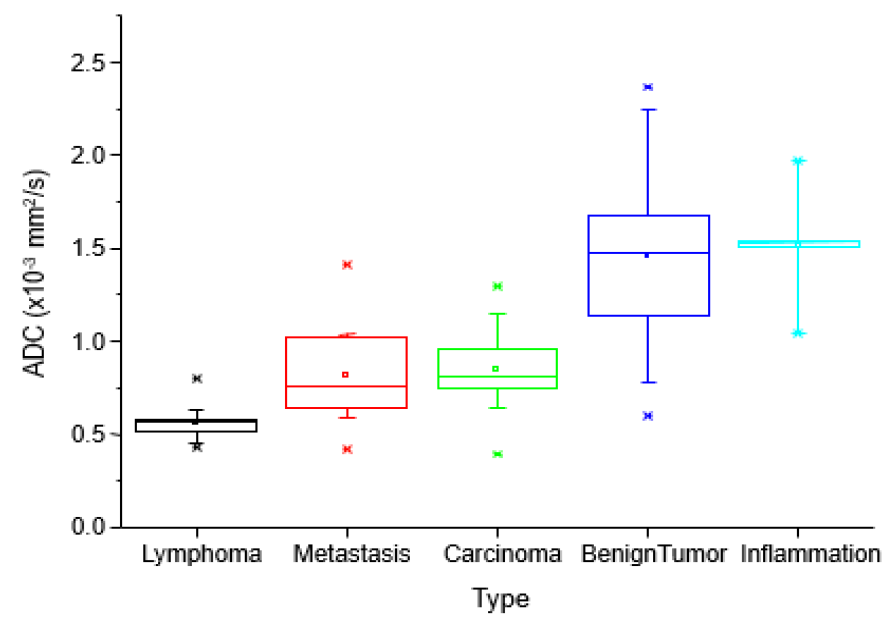

Figure 4. Boxplot showing the distribution of ADC values for different pathologies
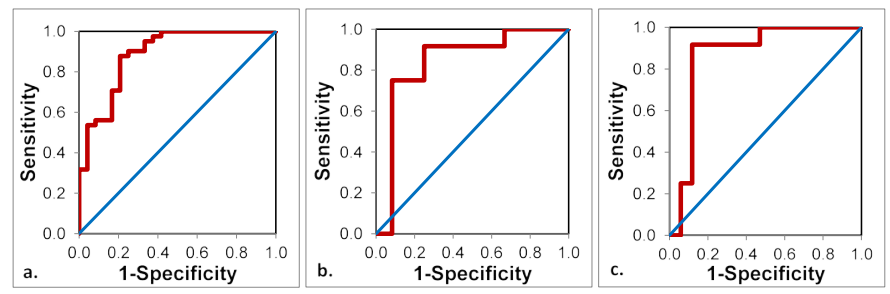

Figure 5. ROC-Curves for the ADC-values for the discrimination between

a. malignant and benign tumors

b. lymphomas and metastases

c. lymphomas and primary carcinomas

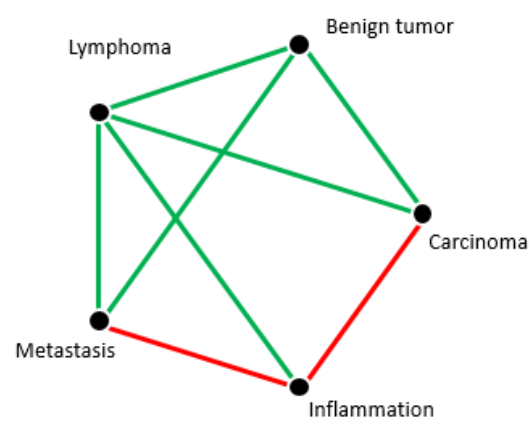

Figure 6. Paired comparison between the different entities

Green lines: significant statistical differences after Bonferroni correction for multiple comparisons

Red lines: significant statistical differences before Bonferroni correction for multiple comparisons

Absence of lines: No statistical difference
Table 2. Paired comparisons between the different entities with the respective p-values

\begin{tabular}{|c|c|c|}
\hline \multicolumn{2}{|c|}{ Entities } & p-value \\
\hline Lymphoma & Metastasis & $\mathbf{0 . 0 4 7}$ \\
\hline Lymphoma & Carcinoma & $\mathbf{0 . 0 0 9 0}$ \\
\hline Lymphoma & Benign Tumor & $\mathbf{0 . 0 0 0 0 3 6}$ \\
\hline Lymphoma & Inflammation & $\mathbf{0 . 0 3 6}$ \\
\hline Metastasis & Carcinoma & 1 \\
\hline Metastasis & Benign Tumor & $\mathbf{0 . 0 0 2 9}$ \\
\hline Metastasis & Inflammation & 0.053 \\
\hline Carcinoma & Benign Tumor & $\mathbf{0 . 0 0 3 5}$ \\
\hline Carcinoma & Inflammation & 0.055 \\
\hline Benign Tumor & Inflammation & 1 \\
\hline
\end{tabular}

- bold: statistically significant

- normal: no statistical significance

for the analysis between lymphoma and metastasis 0.84 , suggesting a good quality for both analyses. The pairwise comparisons are shown in Figure 6 and the corresponding $\mathrm{p}$-values in Table 2.

\section{Discussion}

The results of the current study show that DW-MRI can differentiate between benign lesions, inflammation and malignant tumors. Visual assessment of the diffusion-weighted sequences alone proved to be very effective in detection of malignant lesions, as seen in previous studies [21-26]. However, visual assessment is subjective, is highly dependent on the readers' experience and lastly, comparison with other studies is difficult. ADC values on the other hand are quantitative, and therefore objective, allowing comparison with other studies.

Qualitative analysis proved to be very useful in differentiation between inflammatory lesions and malignant tumors as most inflammatory lesions did not show restricted diffusion and malignant tumors mostly did. In this study, the differences in ADC values between inflammatory and malignant lesions were not statistically significant after conservative Bonferroni correction, most likely due to the small number of subjects in the inflammation group. Still, a clear trend towards higher ADC values in inflammatory lesions was seen compared to the malignant lesions of the head and neck area.

Based on ADC values, lymphomas could be easily differentiated from inflammatory lesions in a statistically significant way. This finding is in keeping with the work of Sepahdari et al., who compared ADC values of lymphomas to inflammatory pseudotumors of the orbit and pointed out significant differences between these two entities [20]. This can be easily explained by the hypercellularity of lymphomas limiting diffusion and the relative hypocellularity of edematous inflammatory lesions allowing more diffusion. Lymphomas could furthermore be clearly differentiated from the other benign and malignant lesions as well, which is also well in keeping with the literature [22-26]. Although a decrease in the ADC values in fungus infection of the masticator space has been reported in literature [21], we did not have any such case in this patient collective. However it should be kept in mind and this highlights the point that diffusion weighted imaging should always be interpreted in conjunction with the other MRI images and the clinical context.

A ROC analysis to determine cut-off values to differentiate between inflammatory and malignant lesions could unfortunately not be performed due to the small sample size of inflammatory lesions. Nevertheless, ROC analysis revealed an optimal threshold value for the differentiation between malignant and all benign lesions, including inflammatory and miscellaneous lesions, to be $1.03 \times 10^{-3} \mathrm{~mm}^{2} / \mathrm{sec}$. This 
Peters AA (2018) The value of diffusion-weighted MRI (DW-MRI) as a tool to differentiate between various tumors and inflammatory diseases in the head and neck region

is highly concordant with the results of Sepahdari et al. who claimed a value $1.0 \times 10^{-3} \mathrm{~mm}^{2} / \mathrm{sec}$ to be the optimal cut-off value for this matter [20] and is also in agreement with most of the pre-existing data [22-28].

The most important limitation of this study is the small sample size of the inflammation group, since CT is still primarily used for imaging complications of inflammation in the head and neck area. Still, a strong trend towards higher ADC values in inflammatory lesions could be seen before Bonferroni corrections.

Another limitation to this study was the inclusion of different imaging protocols with variable b- values. However, despite this limitation, statistically significant results were obtained as mentioned above, consistent with results from previous studies. Thus, it is probable that a consistent DW-MRI protocol, ideally including more b-values and allowing the separation of diffusion and perfusion contributions, would result in even stronger differences between the groups.

Lastly, although most benign tumors are usually relatively hypocellular and malignant lesions are usually hypercellular, a few benign tumors can show diffusion restriction, e.g. Warthin tumors due to epithelial and lymphoid stroma with microscopic slitlike cysts containing proteinous fluid [29]. Therefore, biopsies or fine needle aspirations cannot always be avoided. The use of advanced diffusion weighted imaging, with new post-processing methods such as diffusion kurtosis imaging, diffusion tensor imaging or diffusion tensor tractography [30] has the potential to better characterize the different types of head and neck lesions compared with the whole-lesion mean ADC values.

In summary, our results show that qualitative analysis of diffusionweighted imaging is highly accurate in the differentiation between benign and malignant pathologies in the head and neck area and should be performed routinely. Despite the differences in the imaging protocols, this study shows that there were significant differences in the $\mathrm{ADC}$ values between the various pathologies and these results are concordant with most publications regarding this matter despite the fact that examinations with variable b-values were included in the study.

There is a clear trend towards higher ADC values in inflammatory lesions compared to malignant lesions, but this was not statistically significant after Bonferroni correction for multiple comparisons. It will be the task of upcoming studies to validate the observed trends by including a higher number of inflammatory lesions and also incorporating advanced diffusion imaging, which has the potential to further improve characterization of different types of lesions.

\section{Conflicts of interest}

We confirm that there is no conflict of interest associated with this publication, there has been no financial support for this work that could have influenced its outcome.

\section{Declaration of sole submission to clinical and medical investigations}

We declare that this manuscript is original, has not been published before and is currently not being considered for publication elsewhere.

\section{References}

1. Takahara T, Kwee TC (2012) Low b-value diffusion-weighted imaging: emerging applications in the body. Journal of Magnetic Resonance Imaging 35: 1266-1273.

2. Cui Y, Zhang XP, Sun YS, Tang, L, Shen, L (2008) Apparent diffusion coefficient: potential imaging biomarker for prediction and early detection of response to chemotherapy in hepatic metastases. Radiology 248: 894-900.
3. Dzik-Jurasz A, Domenig C, George M, Wolber J, Padhani A, et al. (2002) Diffusion MRI for prediction of response of rectal cancer to chemoradiation. Lancet 360: 307308. [Crossref]

4. Moffat BA, Chenevert TL, Meyer CR et al (2006) The functional diffusion map: an imaging bio- marker for the early prediction of cancer treatment outcome. Neoplasia 8: 259-267.

5. Sharma U, Danishad KK, Seenu V, Jagannathan NR (2009) Longitudinal study of the assessment by MRI and diffusion-weighted imaging of tumor response in patients with locally advanced breast cancer undergoing neoadjuvant chemotherapy. NMR Biomed 22: $104-113$

6. Yoshino N, Yamada I, Ohbayashi N (2001) Salivary glands and lesions: evaluation of apparent diffusion coefficients with split-echo diffusion-weighted MR imaging- initial results. Radiology 221: 837-842.

7. Namimoto T, Yamashita Y, Mitsuzaki K, Nakayama Y, Tang Y, Takahashi M (1999). Measurement of the apparent diffusion coefficient in diffuse renal disease by diffusionweighted echoplanar MR imaging. J Magn Reson Imaging 9: 832-837.

8. Schafer J, Srinivasan A, Mukherji S (2011) Diffusion magnetic resonance imaging in the head and neck. Magn Reson Imaging Clin N Am 19: 55-67. [Crossref]

9. Thoeny HC, De Keyzer F, Claus FG, Sunaert S, Hermans R (2005) Gustatory stimulation changes the apparent diffusion coefficient of salivary glands: initial experience. Radiology 235: 629-634.

10. Tshering Vogel DW, Zbaeren P, Geretschlaeger A, Vermathen P, De Keyzer F, et al. (2013) Diffusion weighted MR imaging including biexponential for the detection of recurrent or residual tumor after chemo (radiotherapy) for laryngeal and hypopharyngeal cancers. European Journal of Radiology 23: 562-569.

11. King AD, Thoeny HC (2016) Functional MRI for the prediction of treatment response in head and neck squamous cell carcinoma: potential and limitations. Cancer Imaging 16: 23. [Crossref]

12. Thoeny HC (2011) Diffusion-weighted MRI in head and neck radiology: applications in oncology. Cancer Imaging 10: 209-214. [Crossref]

13. Wang J, Takashima S, Takayama F, Kawakami S, Saito A, et al. (2001) Head and neck lesions: characterization with diffusion-weighted echo-planar MR imaging. Radiology 220: 621-630. [Crossref]

14. Wu Q, Zheng D, Shi L, Liu M, Wang M, et al. (2017) Differentiating metastatic from nonmetastatic lymph nodes in cervical cancer patients using monoexponential, biexponential, and stretched exponential diffusion-weighted MR imaging. Eur Radiol 27: 5272-5279. [Crossref]

15. Chen GX, Wang MH, Zheng T, Tang GC, Han FG, Tu GJ (2017) Diffusion-weighted magnetic resonance imaging for the detection of metastatic lymph nodes in patients with lung cancer: A meta-analysis. Mol Clin Oncol 6: 344-354.

16. Wendl CM, Müller S, Eiglsperger J, Fellner C, Jung EM, et al. (2016) Diffusionweighted imaging in oral squamous cell carcinoma using 3 Tesla MRI: is there a chance for preoperative discrimination between benign and malignant lymph nodes in daily clinical routine?. Acta Radiol 57: 939-946.

17. Takahara T, Kwee TC (2017) Diffusion-weighted imaging in quiescent Crohn's disease: correlation with inflammatory biomarkers and video capsule endoscopy. Journal of Magnetic Resonance Imaging 35: 1266-1273.

18. Barendregt AM, van Gulik EC, Lavini C, Nusman CM (2017) Diffusion-weighted imaging for assessment of synovial inflammation in juvenile idiopathic arthritis: a promising imaging biomarker as an alternative to gadolinium-based contrast agents. European Journal of Radiology 43: 239-311.

19. Koç O, Paksoy Y, Erayman I, Kivrak AS, Arbag H (2007) Role of diffusion weighted MR in the discrimination diagnosis of the cystic and/or necrotic head and neck lesions. European Journal of Radiology 62:205-213.

20. Sepahdari AR, Politi LS, Aakalu VK, Kim HJ, Abdel Razek AAK (2014) DiffusionWeighted Imaging of Orbital Masses: Multi-Institutional Data Support a 2-ADC Threshold Model to Categorize Lesions as Benign, Malignant or Indeterminate. American Journal of Neuroradiology 35: 170-175.

21. Abdel Razek AAK, Nada N (2013) Role of diffusion-weighted MRI in differentiation of masticator space malignancy from infection. Dentomaxillofac Radiol 42: 20120183.

22. Abdel Razek AAK, Soliman NY, Elkhamary S, Alsharaway MK, Tawfik A (2006) Role of diffusion-weighted MR imaging in cervical lymphadenopathy. European Radiology 16: $1468-1477$.

23. Holzapfel K, Duetsch S, Fauser C, Eiber M, Rummeny EJ, et al. (2009) Value of diffusion-weighted MR imaging in the differentiation between benign and malignant cervical lymph nodes. European Journal of Radiology 72: 381-387. 
Peters AA (2018) The value of diffusion-weighted MRI (DW-MRI) as a tool to differentiate between various tumors and inflammatory diseases in the head and neck region

24. Vandecaveye V, De Keyzer F, Vander Poorten V, et al. (2009) Head and neck squamous cell carcinoma: value of diffusion-weighted MR imaging for nodal staging. Radiology 251: 134-146.

25. Si J, Huang S, Shi H, Liu Z, Hu Q, et al. (2014) Usefulness of 3T diffusion-weighted MRI for discrimination of reactive and metastatic cervical lymph nodes in patients with oral squamous cell carcinoma: a pilot study. Dentomaxillofac Radiol 43: 20130202. [Crossref]

26. Sumi M, Sakihama N, Sumi T (2003) Discrimination of metastatic cervical lymph nodes with diffusion-weighted MR imaging in patients with head and neck cancer. AJNR Am J Neuroradiol 24: 1627-1634.
27. King AD, Ahuja AT, Yeung DK (2007) Malignant cervical lymphadenopathy: diagnostic accuracy of diffusion-weighted MR imaging. Radiology 245: 806-813.

28. Maeda M, Kato H, Sakuma H, Maier SE, Takeda K (2005) Usefulness of the apparent diffusion coefficient in line scan diffusion-weighted imaging for distinguishing between squamous cell carcinomas and malignant lymphomas of the head and neck. American Journal of Neuroradiology 26: 1186-1192.

29. Abdel Razek AAK (2010) Diffusion weighted magnetic resonance imaging of the head and neck. J Comput Assist Tomogr 34: 808-815.

30. Abdel Razek AAK (2018) Routine and Advanced Diffusion Imaging Modules of the Salivary Glands. Neuroimag Clin N Am 28: 245-254.

Copyright: (92018 Peters AA. This is an open-access article distributed under the terms of the Creative Commons Attribution License, which permits unrestricted use, distribution, and reproduction in any medium, provided the original author and source are credited. 